\title{
LIE TRIPLE DERIVATIONS AND BOSONIC AND FERMIONIC REPRESENTATIONS FOR FILIFORM LIE ALGEBRA $L_{N}$
}

\section{HENGTAI WANG and CHONGHUI HUANG}

School of Mathematics and Physics

University of South China

Hengyang 421001

Hunan

P. R. China

e-mail:wht-2001@163.com

\begin{abstract}
The aim of this paper is to present the decomposition of Lie triple derivations for filiform Lie algebra $L_{N}$. Meanwhile its bosonic and fermionic representations are also constructed.
\end{abstract}

\section{Introduction and Preliminaries}

Let $L$ be a Lie algebra over a commutative ring $R$. Recall that a linear mapping $\phi$ from $L$ to $L$ is called a Lie derivation of $L$, if it satisfies

$$
\phi([x, y])=[\phi(x), y]+[x, \phi(y)], \text { for all } x, y \in L .
$$

2010 Mathematics Subject Classification: 17B30, 17B40.

Keywords and phrases: Lie triple derivation, filiform Lie algebra.

Project supported by the Foundation of University of South China (2011XQD53), Hunan

Provincial Natural Science Foundation of China (Grant 13JJ4075, 14JJ3099), and NSFC (Grant 11126169, 11201220).

Received November 15, 2014

(C) 2015 Scientific Advances Publishers 
It is called a Lie triple derivation (in the paper [1], it is called prederivation), if the following equation holds:

$\phi([[x, y], z])=[[\phi(x), y], z]+[[x, \phi(y)], z]+[[x, y], \phi(z)]$, for all $x, y, z \in L$.

Lie derivations are important research objects in the structure theory for Lie algebras. It is well-known that any Lie derivation for finite dimensional semisimple Lie algebras is inner. So people focused on those for solvable and nilpotent ones [10, 12]. Lie triple derivation, as a natural generalization of Lie derivations, also attracted many authors' attentions $[8,13,14]$. Meanwhile, Lie triple system on infinite-dimensional case is also studied by Martín and González in [9].

Define a decreasing central series for a finite dimensional Lie algebra $L$ as follows:

$$
L^{0}=L, \quad L^{1}=[L, L], \cdots, L^{i+1}=\left[L, L^{i}\right], \cdots .
$$

If there exists a positive integer $s$ such that $L^{s}=0$, then $L$ is called a nilpotent Lie algebra. Filiform Lie algebras are a class of important nilpotent Lie algebras, which were first introduced by Vergne in [11]. They are defined as follows:

Definition 1. A Lie algebra $L$ with $\operatorname{dim} L=n$ is called filiform, or a filiform Lie algebra, if

$$
\operatorname{dim} L^{k}=n-k-1, \quad k \geq 1 .
$$

Here we give four examples of filiform Lie algebras. Actually there are some other types [7]. By [5], any filiform Lie algebra is a deformation of $L_{N}$. So $L_{N}$ has a role importance in filiform Lie algebras. Here the non displayed commutators are either zero or obtained by skew symmetry.

(1) Let $L_{N}$ be the $n$-dimensional Lie algebra with basis $\left\{e_{1}, e_{2}, \cdots, e_{N}\right\}$ satisfying the following brackets:

$$
\left[e_{1}, e_{i}\right]=e_{i+1}, \quad 2 \leq i \leq N-1 .
$$


The undefined brackets are zeros. One can check that it is indeed a filiform Lie algebra. It is, in a certain manner, the simplest filiform Lie algebra.

(2) Let $Q_{n}(n=2 k+1)$ be the $n+1$-dimensional Lie algebra with basis $\left\{X_{0}, X_{1}, \cdots, X_{n}\right\}$. The nonzero Lie brackets are

$$
\begin{aligned}
& {\left[X_{0}, X_{i}\right]=X_{i+1}, \quad 1 \leq i \leq n-1 ;} \\
& {\left[X_{i}, X_{n-i}\right]=(-1)^{i} X_{n}, \quad 1 \leq i \leq k .}
\end{aligned}
$$

This Lie algebra is also filiform.

(3) Let $R_{n}$ be defined in the basis $\left\{X_{0}, X_{1}, \cdots, X_{n}\right\}$ by

$$
\begin{aligned}
& {\left[X_{0}, X_{i}\right]=X_{i+1}, \quad 2 \leq i \leq n-1 ;} \\
& {\left[X_{1}, X_{j}\right]=X_{j+2}, \quad 2 \leq j \leq n-2 .}
\end{aligned}
$$

It is evidently filiform.

(4) Let $W_{n}$ be the Lie algebra whose brackets defined in the basis $\left\{X_{0}, X_{1}, \cdots, X_{n}\right\}$ are

$$
\begin{aligned}
& {\left[X_{0}, X_{i}\right]=X_{i+1}, \quad 2 \leq i \leq n-1 ;} \\
& {\left[X_{i}, X_{j}\right]=\frac{6(i-1) !(j-1) !(j-i)}{(i+j) !} X_{i+j+1}, \quad 1 \leq i<j \leq n-1, i+j+1 \leq n .}
\end{aligned}
$$

This Lie algebra is filiform. It is isomorphic to the Lie algebra defined by

$$
\left[Y_{i}, Y_{j}\right]=(j-i) Y_{i+j}, i+j \leq n+1,1 \leq i<j \leq n-1,
$$

which is a finite quotient of the nilpotent part of Witt algebra.

Significant researches of bosonic and fermionic representations have been done on Kac-Moody algebras, affine Lie algebras, and other infinitely dimensional Lie algebras. For instance, fermionic representations for the affine Kac-Moody algebras were first given by Frenkel [3]. The representations for classical affine Lie algebras were constructed by Feingold and Frenkel [4] by using Clifford or Weyl 
algebras with infinitely many generators. Chen and Gao [2] developed the bosonic and fermionic representations for a class of $B C_{N}$-graded Lie algebras. However, bosonic and fermionic representations have not been given for filiform Lie algebras, so the other aim of this paper is to construct the bosonic and fermionic representations of $L_{N}$.

Throughout this paper, $\operatorname{span}\left\{v_{1}, v_{2}, \cdots, v_{n}\right\}$ stands for the space spanned by $\left\{v_{1}, v_{2}, \cdots, v_{n}\right\}$. The symbols $\mathbb{C}, \mathbb{Z}_{+}$, and $\mathbb{Z}_{-}$denote the set of complex, positive integers, and negative integers, respectively.

\section{Lie Triple Derivations of $L_{N}$}

In this section, we shall fix our discussion on the complex number field $\mathbb{C}$ and give the decomposition of any Lie triple derivation of $L_{N}$. Before this we define four types of standard Lie triple derivations.

(1) For any $x \in L_{N}, a d x(y)=[x, y]$ defines a Lie derivation (also a Lie triple derivation), which is called the Lie triple derivation induced by $x$.

(2) Let $\alpha$ be a linear mapping on $L_{N}$ and defined as

$$
\alpha\left(e_{1}\right)=k_{1} e_{1}+k_{1}^{\prime} e_{2} .
$$

For even $i, \alpha\left(e_{i}\right)=(i-2) k_{1} e_{1}+k_{2} e_{2}$.

For odd $i$ and $i \geq 3, \alpha\left(e_{i}\right)=(i-3) k_{1} e_{1}+k_{3} e_{3}$. Here $k_{1}, k_{1}^{\prime}, k_{2}, k_{3} \in \mathbb{C}$. One can check that $\alpha$ is a Lie triple derivation of $L_{N}$.

(3) For any integer $j \geq 1$ and $l \geq-1, l \neq 0$, define linear mappings $s_{j}$ and $t_{l}$,

$$
\begin{gathered}
s_{j}, t_{l}: L_{N} \rightarrow L_{N}, \\
s_{j}\left(e_{i}\right)=\varepsilon_{j} e_{i+j}, \quad t_{l}\left(e_{i}\right)=\epsilon_{l} e_{i+l} .
\end{gathered}
$$


If $i$ is even and $i+j \leq N, \varepsilon_{j}$ is a nonzero constant; otherwise 0 . If $i$ is odd, $i \geq 3$ and $i+l \leq N, \epsilon_{l}$ is a nonzero constant; otherwise 0 .

Obviously $s_{j}$ 's and $t_{l}$ 's are all Lie triple derivations on $L_{N}$.

(4) For $a_{1}, a_{2}, b_{1}, b_{2} \in \mathbb{C}$, let $\beta$ be a linear mapping of $L_{N}$ defined as follows:

$$
\left\{\begin{array}{l}
\beta\left(e_{2}\right)=a_{1} e_{N-1}+a_{2} e_{N} \\
\beta\left(e_{3}\right)=b_{1} e_{N-1}+b_{2} e_{N} \\
\beta\left(e_{i}\right)=0, \text { otherwise. }
\end{array}\right.
$$

It is not difficult to verify that $\beta$ is a Lie triple derivation of $L_{N}$.

In order to get the decomposition of $\phi$, we assume that

$$
\phi\left(e_{i}\right)=\sum_{j=1}^{N} a_{j}^{(i)} e_{j}
$$

where $a_{j}^{(i)} \in \mathbb{C}$, for all $i, j=1,2, \cdots, N$.

Lemma 1. For any Lie triple derivation $\phi$ of $L_{N}$, we have

$$
\phi\left(e_{i}\right) \in \begin{cases}\operatorname{span}\left\{e_{1}, e_{2}, \cdots, e_{N}\right\}, & i=1 ; \\ \operatorname{span}\left\{e_{i}, e_{i+1}, \cdots, e_{N}\right\}, & i \text { even } \\ \operatorname{span}\left\{e_{i-1}, e_{i}, \cdots, e_{N}\right\}, & i \text { odd } \text { and } i \geq 3 .\end{cases}
$$

Proof. Suppose $\phi$ is an arbitrary Lie triple derivation. By applying $\phi$ on the both sides of $\left[e_{1},\left[e_{2}, e_{3}\right]\right]=0$, we have

$$
\begin{aligned}
0 & =\phi\left(\left[e_{1},\left[e_{2}, e_{3}\right]\right]\right) \\
& =\left[e_{1},\left[\phi\left(e_{2}\right), e_{3}\right]\right]+\left[e_{1},\left[e_{2}, \phi\left(e_{3}\right)\right]\right. \\
& =a_{1}^{(2)} e_{5}-a_{1}^{(3)} e_{4} .
\end{aligned}
$$


So $a_{1}^{(2)}=a_{1}^{(3)}=0$. For any $2 \leq i \leq N-2$, by considering the operation of $\phi$ on $\left[e_{1},\left[e_{1}, e_{i}\right]\right]=e_{i+2}$, we have the desired result.

We now give the theorem of decomposition for any Lie triple derivation of $L_{N}$.

Theorem 1. If $N \geq 6$, any Lie triple derivation $\phi$ of $L_{N}$ can be uniquely expressed as

$$
\phi=a d x+\alpha+\beta+\sum_{j=3}^{N-2} s_{j}+\sum_{\substack{l=2 \\ l \neq 3}}^{N-2} t_{l} .
$$

Proof. By (2.1), we can choose $x=-\sum_{i=2}^{N-1} a_{i+1}^{(1)} e_{i}$, then $(\phi-a d x)\left(e_{1}\right)=$ $a_{1}^{(1)} e_{1}+a_{2}^{(1)} e_{2}$ and $e_{i}(2 \leq i \leq N)$ are stable under $a d x$. Denote $\phi_{1}=\phi-a d x$.

For any even $i$, by applying $\phi_{1}$ on $\left[e_{1},\left[e_{1}, e_{i}\right]\right]=e_{i+2}$, we can get that

$$
\begin{aligned}
& 2 a_{1}^{(1)}+a_{2}^{(2)}=a_{4}^{(4)}, \\
& 2 a_{1}^{(1)}+a_{4}^{(4)}=a_{6}^{(6),}
\end{aligned}
$$

that is to say $(i-2) a_{1}^{(1)}+a_{2}^{(2)}=a_{i}^{(i)}$. Similarly for odd $i$ and $i \geq 3$, we have $(i-3) a_{1}^{(1)}+a_{3}^{(3)}=a_{i}^{(i)}$.

Let $k_{1}=a_{1}^{(1)}, k_{1}^{\prime}=a_{2}^{(1)}, k_{2}=a_{2}^{(2)}$ and denote $\phi_{2}=\phi_{1}-\alpha . \quad$ By applying $\phi_{2}$ on $\left[e_{1},\left[e_{1}, e_{i}\right]\right]=e_{i+2}$, we can show that the following two equalities hold:

$$
\begin{cases}a_{j}^{(2)}=a_{j+2}^{(4)}=a_{j+4}^{(6)}=\cdots, & 3 \leq j \leq N-2 \\ a_{l}^{(3)}=a_{l+2}^{(5)}=a_{l+4}^{(7)}=\cdots, & 2 \leq l \leq N-2, l \neq 3 .\end{cases}
$$


Let $a_{j}^{(2)}=\varepsilon_{j}, a_{l}^{(3)}=\epsilon_{l}$ and $\phi_{3}=\phi_{2}-\sum_{j=3}^{N-2} s_{j}-\sum_{\substack{l=2 \\ l \neq 3}}^{N-2} t_{l}$, then

$$
\left\{\begin{array}{l}
\phi_{3}\left(e_{i}\right) \in \operatorname{span}\left\{e_{N-1}, e_{N}\right\}, \quad i=2,3 \\
\phi_{3}\left(e_{i}\right)=0, \quad \text { otherwise. }
\end{array}\right.
$$

By choosing $a_{1}=a_{N-1}^{(2)}, a_{2}=a_{N}^{(2)}, b_{1}=a_{N-1}^{(3)}, b_{2}=a_{N}^{(3)}$, we can prove that $\left(\phi_{3}-\beta\right)\left(e_{i}\right)=0$, for all $i$. So any Lie triple derivation $\phi$ has the decomposition of (2.2).

Suppose that $\phi=0$, that is to say $\phi\left(e_{i}\right)=0$ for all $i \neq 1,2,3$, we have $s_{j}=t_{l}=0$, if $j+i \leq N$ and $l+i \leq N$. Meanwhile $\varepsilon_{j}$ and $\epsilon_{l}$ are 0 when $j+i>N$ and $l+i>N$. Then all $s_{j}$ and $t_{l}$ are 0 . It follows that $\beta=0$ from $(a d x+\alpha+\beta)\left(e_{2}\right)=(a d x+\alpha+\beta)\left(e_{3}\right)=0$, hence $a d x+\alpha=0$. That $(a d x+\alpha)\left(e_{1}\right)=0$ implies $a d x=0$ and then $\alpha=0$, which completes the proof of uniqueness of the decomposition.

Remark 1. The length of the two equalities in (2.3) is variable. That is to say, if the subscript of $a_{j+2 k}^{(i)}$ are greater than $N$, the equalities will end before this term.

Remark 2. The uniqueness of this decomposition depends on the definitions of standard Lie triple derivations. Once they are defined, the decomposition is unique. In another word, if the definition of $\alpha$ is changed, then those of $s_{j}$ and $t_{l}$ will be also changed, but the types of Lie triple derivations are the same. 


\section{The Representations of $L_{N}$}

In this section, we construct the representations for filiform Lie algebra $L_{N}$ given in Section 1 . Let $A$ be an associative algebra and $\rho= \pm 1$, the $\rho$-bracket is defined as follows:

$$
\{a, b\}_{\rho}=a b+\rho b a, \quad a, b \in A .
$$

It is easy to see that

$$
\{a, b\}_{\rho}=\rho\{b, a\}_{\rho} \text { and }[a b, c]=a\{b, c\}_{\rho}-\rho\{a, c\}_{\rho} b,
$$

for $a, b, c \in A$, where $[a, b]=\{a, b\}_{-1}$ is the Lie bracket.

Define $\mathcal{V}$ to be a unital associative algebra with $2 N$ generators $a_{i}, a_{i}^{*}, 1 \leq i \leq N$ with respect to the relations

$$
\left\{a_{i}, a_{j}\right\}_{\rho}=\left\{a_{i}^{*}, a_{j}^{*}\right\}_{\rho}=0 \text { and }\left\{a_{i}, a_{j}^{*}\right\}_{\rho}=\rho \delta_{i j} .
$$

Let $\mathcal{V}(N, \rho)$ be the unital associative algebra generated by

$$
\left\{u(m) \mid u \in\left(\bigoplus_{i=1}^{N} \mathbb{C} a_{i}\right) \oplus\left(\bigoplus_{i=1}^{N} \mathbb{C} a_{i}^{*}\right), m \in \mathbb{Z}\right\},
$$

with the relations

$$
\{u(m), v(n)\}_{\rho}=\{u, v\}_{\rho} \delta_{m+n, 0} .
$$

We now define the normal ordering as in [4]

$$
: u(m) v(n):=\left\{\begin{array}{l}
u(m) v(n), \quad \text { if } n>m ; \\
\frac{1}{2}(u(m) v(n)-\rho v(n) u(m)), \quad \text { if } m=n \\
-\rho v(n) u(m), \quad \text { if } m>n .
\end{array}\right.
$$

One can check that the following equalities hold:

$$
: a_{i}(m) a_{j}(n):=a_{i}(m) a_{j}(n)=-\rho a_{j}(n) a_{i}(m),
$$




$$
: a_{i}(m) a_{j}^{*}(n):=a_{i}(m) a_{j}^{*}(n)-\rho \delta_{i j} \delta_{m+n, 0} \theta(m-n)
$$

and

$$
\begin{aligned}
& {\left[a_{i}(m) a_{j}(n), a_{k}(p)\right]=0,} \\
& {\left[a_{i}(m) a_{j}(n), a_{k}^{*}(p)\right]=-\delta_{i k} \delta_{m+p, 0} a_{j}(n)+\rho \delta_{j k} \delta_{n+p, 0} a_{i}(m),}
\end{aligned}
$$

where $m, n, p \in \mathbb{Z}, 1 \leq i, j, k \leq N$.

Define $\mathcal{V}(N, \rho)^{+}$to be the subalgebra generated by $a_{i}(n), a_{j}^{*}(m), a_{k}^{*}(0)$, for $n, m \in \mathbb{Z}_{+}$, and $1 \leq i, j, k \leq N$. Let $\mathcal{V}(N, \rho)^{-}$be the subalgebra generated by $a_{i}(n), a_{j}^{*}(m), a_{k}(0)$, for $n, m \in \mathbb{Z}_{-}$, and $1 \leq i, j, k \leq N$. As in $[2,4]$, let $\mathcal{M}(N, \rho)$ be a simple $\mathcal{V}(N, \rho)$-module containing the vacuum vector $v_{0}$, and satisfying

$$
\mathcal{V}(N, \rho)^{+} v_{0}=0
$$

So all annihilation operators kill $v_{0}$ and

$$
M(N, \rho)=\mathcal{V}(N, \rho) v_{0}=\mathcal{V}(N, \rho)^{-} v_{0} .
$$

For $s \in \mathbb{Z}, 1 \leq i \leq N-1$ and any fixed $m \in \mathbb{Z}$, we are now to construct the bosons (if $\rho=-1$ ) or fermions (if $\rho=1$ ) as follows:

$$
\begin{aligned}
f(m) & =\sum_{i=1}^{N-1} \sum_{s \in \mathbb{Z}}: a_{i}(m-s) a_{i+1}^{*}(s): \\
g_{j}(m) & =\sum_{s \in \mathbb{Z}}: a_{1}((j-1) m+s) a_{N+1-j}(m-s) .
\end{aligned}
$$

Theorem 2. Suppose $L_{N}$ is the filiform Lie algebra defined as above with basis $e_{1}, e_{2}, \cdots, e_{N}$. Let

$$
\begin{aligned}
\tau_{m}\left(e_{1}\right) & =f(m), \\
\tau_{m}\left(e_{j}\right) & =g_{j}(m), \quad 2 \leq j \leq N .
\end{aligned}
$$


Then $\tau_{m}$ is a representation of $L_{N}$ on $V(N, \rho)$.

Proof. In view of (3.1), for any $2 \leq j \leq N-1$,

$$
\begin{aligned}
& {\left[f(m), g_{j}(m)\right] } \\
= & {\left[\sum_{i=1}^{N-1} \sum_{s \in \mathbb{Z}}: a_{i}(m-s) a_{i+1}^{*}(s):, \sum_{t \in \mathbb{Z}}: a_{1}((j-1) m+t) a_{N+1-j}(m-t):\right] } \\
= & {\left[\sum_{i=1}^{N-1} \sum_{s \in \mathbb{Z}} a_{i}(m-s) a_{i+1}^{*}(s), \sum_{t \in \mathbb{Z}} a_{1}((j-1) m+t) a_{N+1-j}(m-t)\right] } \\
= & \sum_{i=1}^{N-1} \sum_{s, t \in \mathbb{Z}} a_{i}(m-s)\left[a_{i+1}^{*}(s), a_{1}((j-1) m+t) a_{N+1-j}(m-t)\right] \\
& +\sum_{i=1}^{N-1} \sum_{s, t \in \mathbb{Z}}\left[a_{i}(m-s), a_{1}((j-1) m+t) a_{N+1-j}(m-t)\right] a_{i+1}^{*}(s) \\
= & -\sum_{s \in \mathbb{Z}} \rho a_{N-j}(m-s) a_{1}(j m-s) \\
= & \sum_{s \in \mathbb{Z}}: a_{1}(j m-s) a_{N-j}(m-s): \\
= & g_{j+1}(m) .
\end{aligned}
$$

Similarly, one can show that $\left[f(m), g_{N}(m)\right]=0$.

According to (3.1), for any $i, j \neq 1$, we have

$$
\begin{aligned}
& {\left[g_{i}(m), g_{j}(m)\right]} \\
& =\left[\sum_{s \in \mathbb{Z}}: a_{1}((i-1) m+s) a_{N+1-i}(m-s):, \sum_{t \in \mathbb{Z}}: a_{1}((j-1) m+t) a_{N+1-j}(m-t):\right] \\
& =\left[\sum_{s \in \mathbb{Z}} a_{1}((i-1) m+s) a_{N+1-i}(m-s), \sum_{t \in \mathbb{Z}} a_{1}((j-1) m+t) a_{N+1-j}(m-t)\right]
\end{aligned}
$$




$$
\begin{aligned}
& =\sum_{s, t \in \mathbb{Z}} a_{1}((i-1) m+s)\left[a_{N+1-i}(m-s), a_{1}((j-1) m+t) a_{N+1-j}(m-t)\right] \\
& +\sum_{s, t \in \mathbb{Z}}\left[a_{1}((i-1) m+s), a_{1}((j-1) m+t) a_{N+1-j}(m-t)\right] a_{N+1-i}(m-s) \\
& =0 .
\end{aligned}
$$

For any $m \in \mathbb{Z}$, there is a bosonic or fermionic representation $\pi_{m}$. All of $\pi_{m}$ 's are equivalent mutually. Meanwhile, if we fix an integer $m$, let

$$
\begin{aligned}
& \tilde{f}(m)=\sum_{i=2}^{N} \sum_{s \in \mathbb{Z}}: a_{i-1}(m-s) a_{i}^{*}(s): \\
& \widetilde{g}_{j}(m)=\sum_{s \in \mathbb{Z}}: a_{N}^{*}((j-1) m+s) a_{j-1}^{*}(s):(2 \leq j \leq N),
\end{aligned}
$$

and define

$$
\begin{gathered}
\pi_{m}\left(e_{1}\right)=f(m), \\
\pi_{m}\left(e_{j}\right)=g_{j}(m), \quad 2 \leq j \leq N,
\end{gathered}
$$

then $\pi_{m}$ is also a representation of $L_{N}$.

It is known from [5] that any filiform Lie algebra arises as a linear deformation of the model algebra $L_{N}$. So by Theorem 2 and this linear deformation, we can get the bosonic and fermionic representations of any filiform Lie algebra.

For example, let $P_{N}$ be a Lie algebra with basis $\left\{X_{1}, X_{2}, \cdots, X_{N}\right\}$ subject to the relations

$$
\left[X_{1}, X_{i}\right]=X_{i+1}, \quad 2 \leq i \leq n-1
$$




$$
\left[X_{2}, X_{j}\right]=X_{j+1}, \quad 3 \leq j \leq n-1 .
$$

It is not difficult to verify that this Lie algebra is a deformation of $L_{N}$. In another word, there exists a linear space isomorphism (not Lie isomorphism) $\sigma$ from $P_{N}$ to $L_{N}$, where $\sigma$ is defined by

$$
\sigma\left(X_{2}\right)=e_{1}+e_{2}, \sigma\left(X_{i}\right)=e_{i}, \quad i=1,3,4, \cdots, N
$$

then the bosonic and fermionic representations can be constructed as follows:

$$
\begin{aligned}
& X_{1}=\sum_{i=1}^{N-1} \sum_{s \in \mathbb{Z}}: a_{i}(m-s) a_{i+1}^{*}(s): \\
& X_{2}=\sum_{i=1}^{N-1} \sum_{s \in \mathbb{Z}}: a_{i}(m-s) a_{i+1}^{*}(s):+\sum_{s \in \mathbb{Z}}: a_{1}((j-1) m+s) a_{N-1}(m-s): \\
& X_{j}=\sum_{s \in \mathbb{Z}}: a_{1}((j-1) m+s) a_{N+1-j}(m-s):(3 \leq j \leq N) .
\end{aligned}
$$

\section{References}

[1] D. Burde, Lie algebra prederivations and strongly nilpotent Lie algebras, Comm. in Algebra 30(7) (2002), 3157-3175.

[2] H. Chen and Y. Gao, $B C_{N}$-graded Lie algebras arising from fermionic representations, J. Algebra 308 (2007), 545-566.

[3] I. B. Frenkel, Spinor representations of affine Lie algebras, Proc. Natl. Acad. Sci. 77 (1980), 6303-6306.

[4] A. J. Feingold and I. B. Frenkel, Classical affine algebras, Adv. Math. 56 (1985), 117-172.

[5] J. R. Gómez, A. Jimenéz-Merchán and Y. Khakimdjanov, Low-dimensional filiform Lie algebras, J. Pure Applied Algebra 130 (1998), 133-158.

[6] M. Goze and Y. Khakimdjanov, Nilpotent Lie Algebra, Mathematics and its Applications, The Netherlands: Kluwer Academic Publishers, MIA 361 (1996).

[7] M. Hazewinkel, Handbook of Algebra, Vol. 2, CMI Amsterdam, 2000.

[8] P. Ji and L. Wang, Lie triple derivation of TUHF algebra, Linear Algebra Appl. 403 (2005), 399-408. 
[9] A. J. Martín and C. M. González, The Banach-Lie group of Lie triple automorphisms of an $H^{*}$-algebra, Acta Math. Sci. Ser. B Engl. Ed. 30(4) (2010), 1219-1226.

[10] S. Ou, D. Wang and R. Yao, Derivations of the Lie derivation of strictly upper triangular matrices over a commutative ring, Linear Algebra Appl. 424(2-3) (2007), 378-383.

[11] M. Vergne, Cohomologie des algèbres de Lie nilpotentes, Application à l'étude de la variété des algèbres de Lie nilpotentes', Bull. Soc. Math. 98 (1970), 81-116.

[12] D. Wang and Q. Yu, Derivations of the parabolic subalgebras of the general linear Lie algebra over a commutative ring, Linear Algebra Appl. 416(2-3) (2006), 763-774.

[13] H. Wang and Q. Li, Lie triple derivation of the Lie algebra of strictly upper triangular matrix over a commutative ring, Linear Algebra Appl. 430(1) (2009), 66-77.

[14] J. Zhang and H. Cao, Lie triple derivation of nest algebra, Linear Algebra Appl. 416(2-3) (2006), 559-567. 